\title{
Le pluralisme thérapeutique des migrants et héritiers de l'exil maghrébin en France. Nouvelles données et perspectives
}

Therapeutic Pluralism of Migrants and Heirs of the Maghreb Exile in France.

New Data and Perspectives

El pluralismo terapéutico de los migrantes y descendientes del exilio magrebí en Francia. Nuevos hechos y perspectivas

François Sicot et Slimane Touhami

\section{(2) OpenEdition \\ Journals}

Édition électronique

URL : https://journals.openedition.org/remi/10870

DOI : $10.4000 /$ remi. 10870

ISSN : $1777-5418$

Éditeur

Université de Poitiers

Édition imprimée

Date de publication : 1 octobre 2018

Pagination : 101-130

ISBN : 979-10-90426-62-7

ISSN : 0765-0752

Référence électronique

François Sicot et Slimane Touhami, « Le pluralisme thérapeutique des migrants et héritiers de l'exil maghrébin en France. Nouvelles données et perspectives », Revue européenne des migrations internationales [En ligne], vol. 34 - n² et 3 | 2018, mis en ligne le 01 janvier 2021, consulté le 14 avril 2022. URL : http://journals.openedition.org/remi/10870 ; DOI : https://doi.org/10.4000/remi.10870 


\title{
Le pluralisme thérapeutique des migrants et héritiers de l'exil maghrébin en France. Nouvelles données et perspectives
}

\author{
François Sicot ${ }^{1}$ et Slimane Touhami ${ }^{2}$
}

\section{Introduction}

Comment les migrants du Maghreb et leurs héritiers font-ils face aux troubles psychiques? II convient d'abord de noter que notre question, centrée sur le recours, exclut tout un ensemble de travaux, nombreux, sur la santé mentale des migrants ou sur les soins en santé mentale ailleurs qu'en France et sur d'autres continents.

La question des recours aux soins des migrants a été abordée en France essentiellement sous deux angles. Le premier met l'accent sur le recours comme ensemble des possibilités d'être pris en charge dans le système institutionnel dominant au regard des conditions de vie précaires et du droit - et particulièrement du droit des étrangers, des politiques de santé et de l'immigration. Les travaux qui s'y inscrivent montrent les difficultés d'accéder aux soins, en particulier du fait de discriminations ou de droits spécifiques et restrictifs (Revue Européenne des Migrations Internationales, 2011 et 2012; Hommes \& Migrations, 2000 et 2009; Hoyez, 2015; Gabarro, 2018; Izambert, 2018). La dimension culturelle des pratiques s'en est trouvée ignorée.

Le second angle porte sur ce qu'il convient de faire ou pas pour soigner les migrants. II existe en effet une pluralité de théories et de pratiques professionnelles qui se proposent de les soigner en prenant en compte d'une manière ou d'une autre le bain culturel au sein duquel ils ont été socialisés (Baubet et Moro, 2003). En France, un courant de l'ethnopsychiatrie, développé autour de Nathan, a donné une tournure radicale à cette volonté en créant des dispositifs de soins inspirés des thérapies traditionnelles et en interprétant leurs souffrances et leurs pathologies à partir de la culture d'origine. Cette ethnopsychiatrie a été critiquée pour son culturalisme, c'est-à-dire le fait de postuler l'existence d'un inconscient

1 Sociologue, Professeur, LISST, Université Toulouse 2 Le Mirail, 5 allées A. Machado, 31058 Toulouse cedex 9; sicot@univ-tlse2.fr

2 Anthropologue, formateur-chercheur, CRFMS Erasme, 134 route d'Espagne, BP 53566, 31035 Toulouse cedex 1; stouhami@erasme.fr 
ethnique et de renvoyer systématiquement les migrants à la culture ethnique de leur pays d'origine (Fassin, 2000b; Rechtman, 2000). Plus récemment, Wang a très précisément documenté la diversité des formes de souffrance psychique vécues par les migrants chinois à Paris (2017) en fonction de la vague d'immigration, des causes et des modalités de celle-ci, des conditions de vie passées en Chine et actuelles en France. C'est à partir de cette connaissance qu'elle a entrepris, lors de son enquête participative à des consultations interculturelles, de "démontrer aux médecins la thèse selon laquelle les Chinois n'existent pas culturellement" et qu'il convenait de les "sensibiliser aux facteurs sociaux tels que l'âge, la génération, le genre et la classe - bref, autres que l'origine ethnique - pouvant conduire aux malentendus ou incompréhensions avec les familles chinoises" (Wang, $2016:$ 150).

Explorer la dimension culturelle des pratiques de santé ne doit pas amener à reconduire la notion de culture d'origine qui laisse penser qu'elle constitue un système stable. "On ne transporte pas une culture comme on transporte une valise. Voir les choses comme cela, ce serait tomber dans une réification de la culture, qui n'est en définitive qu'une abstraction. Ce qui se déplace, en réalité, ce sont des individus; et ces individus, du fait même de leur migration, sont amenés à s'adapter et à évoluer» (Cuche, $2012: 49$ ).

\section{Le pluralisme thérapeutique au cœur de différentes traditions de recherche}

Si la vocation de la sociologie ne peut pas être, contrairement à l'ethnopsychiatrie, d'encourager les migrants à retourner dans le pays de leurs ancêtres pour accomplir un rituel et conjurer ainsi le mauvais sort à l'origine de leurs souffrances (Nathan, 1994), elle consiste par contre, à s'interroger sur de possibles recours alternatifs à la biomédecine. Trois courants de recherche permettent de problématiser ces recours : l'ethnologie appliquée à l'étude de la médecine populaire, I'anthropologie de la maladie et les travaux plus récents sur les médecines non conventionnelles. Ces trois ensembles, relativement disjoints, attestent unanimement de la prégnance du pluralisme médical dans les itinéraires thérapeutiques et, loin de le considérer comme une survivance, montrent à l'inverse son étendue, son dynamisme, son renouvellement, en France comme ailleurs.

Les recherches sur les médecines dites non conventionnelles concernent des soins qui s'inscrivent explicitement dans un rapport dialectique et critique avec la biomédecine (Cohen et Rossi, 2011). De ce point de vue, elles correspondent à la définition qu'en donne le ministère de la Santé : "Leur point commun est qu'elles ne sont ni reconnues, au plan scientifique, par la médecine conventionnelle, ni enseignées au cours de la formation initiale des professionnels de santé". Dans le monde anglo-saxon, on les qualifie de complémentaires pour souligner ce qu'elles apportent au patient par rapport à la biomédecine jugée insuffisante ou insatisfaisante (Cant et Sharma, 1999). De par sa construction d'objet, cette littérature ne s'est pas intéressée spécifiquement aux migrants et à ce que leurs pratiques de soins, leurs recours en cas de maladie pourraient devoir à une socialisation culturelle donnée.

S'il n'existe pas de liste exhaustive des médecines conventionnelles, en est exclu tout un ensemble de recours qui relèvent bien du pluralisme médical, mais 
que I'anthropologie a pu nommer traditionnels et qui recouvrent l'ensemble des pratiques qui relèvent $d^{\prime}$ 'un système culturel, religieux dans sa globalité et dans une société donnée. Historiquement, les anthropologues qui se sont intéressés au pluralisme médical ont réalisé leurs terrains dans deux types de sociétés: d'une part celles des pays non occidentaux dans lesquels les anthropologues ont été sollicités pour comprendre et aider les acteurs politiques à diffuser la médecine allopathique malgré les obstacles représentés par les croyances et l'omniprésence des thérapeutiques traditionnelles (Dozon, 1983). D'autre part, les sociétés dans lesquelles la diversité culturelle, le métissage, le brassage culturel et donc le pluralisme médical étaient des évidences. "La notion d'itinéraire thérapeutique met l'accent sur l'ensemble des processus impliqués, avec leurs détours et sinuosités, dans une quête thérapeutique, allant de l'apparition d'un trouble à toutes les étapes, institutionnelles ou non, où peuvent s'actualiser diverses interprétations (divination, rumeur, etc.) et cures, dans des contextes de pluralisme médical " (Sindzingre, 1985 : 14). Très rapidement (Augé et Herzlich, 1987; Benoist, 1996), ces travaux ont montré la présence de l'innovation dans la tradition, et que la tradition n'indiquait pas la permanence d'un passé figé dans le présent (Lenclud, 1987). Parler de médecine traditionnelle c'est reconduire l'opposition binaire entre sociétés traditionnelles/modernes, alors même que, dans le domaine de la santé, la modernité semble être à l'intégration des médecines non conventionnelles et traditionnelles ${ }^{3}$. Dans Les nouveaux guérisseurs, les auteurs montrent l'apparition en Afrique, sur les continents asiatique, américain de guérisseurs d'un nouveau genre dont les pratiques, tout en se référant aux médecines traditionnelles, empruntent divers éléments à des orientations religieuses et médicales contemporaines, donnant lieu ainsi à des "thérapies kaléidoscopiques" ${ }^{4}$. Mais à nouveau, ces travaux laissent de côté la question de savoir si les migrants installés en France recourent, au long de leur itinéraire thérapeutique, à un pluralisme médical qui puiserait à une tradition, même renouvelée.

Les travaux sur les mobilités et la santé dans le cadre de la globalisation décrivent la circulation des médecins et des patients des pays des Suds vers les pays du Nord, mais également des circulations sud-sud ou des recours de patients ou de clients des classes moyennes et supérieures du Nord vers le Sud et ses ressources considérées comme traditionnelles (Pordié, 2011). Mais qui sont les clients ou les patients de ce nouveau pluralisme médical qui émerge de la globalisation? L'article de Hoyez (2012) "L'ayurveda, c'est pour les Français" -, pourrait être de ce point de vue illustratif de l'idée selon laquelle, le pluralisme médical concerne une part non négligeable de la population, mais pas les migrants. L'auteure commence en effet ainsi son article : "Les études sur les démarches de santé des populations migrantes évoquent couramment la recherche et/ou l'usage de pratiques de soins ne relevant pas du système biomédical (Gronseth et Oakley, 2007 ; The Franco-British Working Group on Migration, Health and Wellbeing, 2009 ; Thomas, 2010). Elles sous-entendent ou avancent explicitement que, dans la migration, les individus transfèreraient un ensemble

3 La Stratégie de I'OMS pour la médecine traditionnelle pour 2014-2023 se donne comme objectif "d'intégrer la médecine traditionnelle et la médecine complémentaire et parallèle aux systèmes nationaux de soins de santé, de manière appropriée».

4 La postface de cet ouvrage, rédigée par Fassin est titrée La tradition, toujours réinventée. 
de connaissances relatives à la santé, empruntées aux savoirs médicaux élaborés dans les pays d'origine, et organisées au sein de réseaux transnationaux liant des patients et des praticiens " (2012: 149). II faut pourtant remarquer qu'il n'y a pas de telles études en France, et que Hoyez s'inscrit dans la seule perspective qui y soit développée et qui pointe du doigt le culturalisme de telles approches et souligne, a contrario, les "facteurs individuels et collectifs, sociaux et politiques qui, en se conjuguant, influent sur les logiques de recours aux soins des populations migrantes" (Hoyez, 2012 : 149).

Enfin, un troisième ensemble de recherches montre la présence du pluralisme médical en France. S'inscrivant dans les pas de l'œuvre des folkloristes, l'ethnologie de la médecine populaire étudie les objets, recettes, prières, légendes des guérisseurs et autres leveurs de maux des "campagnes" (Loux, 1974; Laplantine, 1978; Favret-Saada, 1977). Des travaux plus récents montrent que le recours aux guérisseurs et autres praticiens luttant contre la sorcellerie se poursuit, se renouvelle et soit $s^{\prime}$ inscrit dans un habitus familial et un système de soins local (Raineau, 2002; Lemonnier, 2016), soit vient puiser dans la science contemporaine en évoquant la présence d'ondes, d'énergies positives ou négatives, en proposant des reformulations chamaniques (Schmitz, 2006) ou issues de la tradition catholique et d'autres religions (Kessler-Bilthauer, 2018).

Faut-il conclure de cette rapide revue de littérature que les migrants présents en France ne sont pas concernés par le pluralisme culturel au sein de leurs itinéraires thérapeutiques, en particulier sous la forme d'un recours à des soins traditionnels présents dans leur société d'origine? Sous réserve d'oublis, on peut affirmer que quelques travaux seulement font exception et qu'il n'existe de toute façon pas de recherches en nombre suffisamment important, se cumulant et se discutant pour aboutir à une connaissance du phénomène. Epelboin (1996) et Kuczynski (2008) ont fait état des pratiques de maraboutage d'Africains à Paris, en particulier en présence de maladie, ainsi que de l'importance des voyages en Afrique soit du malade lui-même soit de sa famille pour y trouver une aide supplémentaire. Cherak (2013) s'est intéressée spécifiquement à la roqya ou "médecine du prophète" et à ses traitements axés sur le retour à l'islam traditionnel.

Notre article prolonge des travaux que nous avons menés antérieurement et porte ici sur ce que Kleinman (1980) nomme le secteur traditionnel du système de soins. À propos des comportements de recherche de soins ou des stratégies de recours aux soins, cet auteur considère que les individus s'orientent dans des systèmes de soins qui sont composés de trois secteurs qui se chevauchent: les secteurs populaire, professionnel et traditionnel. C'est le système dans sa totalité qui soigne. Dans chaque secteur, la maladie est perçue, nommée et interprétée et un type spécifique de soins est appliqué. Dans le secteur populaire, l'individu est en interaction avec son entourage proche, non spécialisé. C'est le lieu de l'identification du trouble et de l'évaluation de ses retentissements par I'individu et par sa famille. Lieu de l'auto-soin, il est aussi celui des conduites préventives et de protection. Dans sa thèse d'ethnologie sur le Maghreb de France, l'un d'entre nous (Touhami, 2010) explorait ce secteur populaire au travers de la description des pratiques quotidiennes de protection du mauvais œil, leur transmission, leurs transformations, au sein de familles de migrants et de leurs enfants. II y montrait la persistance, dans de nombreux foyers, de 
pratiques traditionnelles de médication ainsi que la croyance en diverses catégories d'êtres invisibles et, plus globalement, les représentations traditionnelles du "désordre" dans la diaspora maghrébine.

\section{Méthodologie}

Dans une recherche ultérieure (Sicot et Touhami, 2015) menée auprès de cinquante soignants en psychiatrie exerçant sur trois sites, nous avons commencé à explorer le secteur professionnel dominant, celui de la biomédecine. En les interrogeant avec un focus très précis sur la place qu'ils accordaient à la culture de leurs patients d'origine maghrébine dans des soins, ils ont été nombreux à citer l'existence d'une diversité de pratiques - retour au pays d'émigration, fumigations, lectures du Coran, incantations, évitement d'aliments supposés être dangereux, etc. - sans qu'il leur soit possible de préciser ces pratiques et leur ampleur.

Afin d'avoir une vision complète du système de soins dans lequel évoluent les patients d'origine maghrébine en France, nous avons donc entrepris de mener l'enquête auprès des patients eux-mêmes et de ce que Kleinman nomme le secteur traditionnel, formé des spécialistes non professionnels. Outre que nous reprenons les termes de Kleinman et que nous avons cité plus haut un certain nombre de précautions à prendre lorsqu'on utilise le terme de traditionnel, on ajoutera que les pays du Maghreb sont devenus des pays d'immigration, en particulier d'Afrique subsaharienne (Bensaâd, 2009), produisant un nouveau cosmopolitisme (Khrouz et Lanza, 2015). Et tandis que les patients originaires du Maghreb se réfèrent à des systèmes d'interprétation issus de leur pays d'origine, les professionnels de la médecine moderne recourent de leur côté à diverses thérapies anciennes et se forment à la médecine intégrée ${ }^{5}$.

Quatorze entretiens ont été réalisés àToulouse auprès de patients de psychiatrie maghrébins (Maroc, Tunisie, Algérie) installés en France depuis des durées variables. Onze sont de nationalité française de naissance ou par naturalisation. Tous, ont des parents nés au Maghreb (Tableau 1). Le terme "d'héritier " utilisé pour les qualifier permet d'indiquer cette ascendance en soulignant l'aspect dynamique d'un rapport aux héritages dont les contenus sont susceptibles d'être revisités, en fonction de choix ou de conditions de vie. II permet d'éviter le non-sens de la désignation d'une "énième " génération d'immigrés ou à une origine supposée sur la base de critères ethnoculturels non objectivables. Les patients ont été recrutés dans des structures de soins psychiatriques (centres médico-psychologiques, hôpital de jour, permanence d'accès aux soins ${ }^{6}$ ) et

5 Le terme de médecine intégrée est utilisé pour décrire les soins de santé où la médecine complémentaire et alternative (CAM) est prise à l'intérieur du modèle biomédical. On peut citer par ailleurs le développement de la médecine chinoise dans les grandes villes de Tunisie, du Maroc et de l'Algérie.

6 Présent sur chaque secteur de psychiatrie, le CMP assure des consultations pour toute personne en situation de souffrance psychique et organise leur éventuelle orientation vers des structures adaptées. L'hôpital de jour accueille pour sa part en ambulatoire des malades souffrant de troubles psychiques qui nécessitent des soins quotidiens et prolongés. Il existe des hôpitaux de jour pour adultes et d'autres spécialisés dans la prise en charge pour enfants et adolescents. Enfin, la Permanence d'Accès aux Soins de Santé (PASS) accueille, écoute et oriente toute personne présentant un problème de santé et de couverture sociale. II existe à Toulouse une PASS psychiatrie dépendant du CHU local. 
Tableau 1 : Principales caractéristiques des personnes interrogées

\begin{tabular}{|c|c|c|c|c|c|c|c|c|c|c|c|}
\hline E & ڤ゙્ & $\stackrel{0}{\circ}$ & 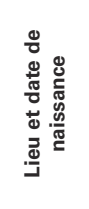 & 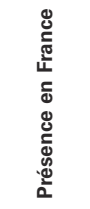 & 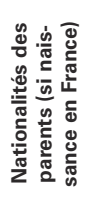 & 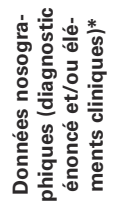 & 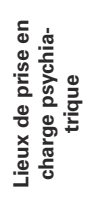 & 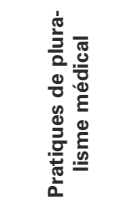 & 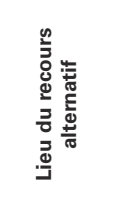 & 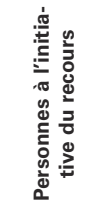 & 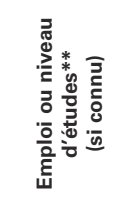 \\
\hline Amar & $\mathrm{H}$ & 39 & $\begin{array}{c}\text { Algérie } \\
1974\end{array}$ & $\begin{array}{c}1999- \\
2001 \\
\text { [expul- } \\
\text { sion] } \\
2011 \rightarrow\end{array}$ & & $\begin{array}{l}\text { Dépres- } \\
\text { sion, } \\
\text { tentative } \\
\text { de suicide }\end{array}$ & $\begin{array}{l}\text { Suivi } \\
\text { CMP }\end{array}$ & Fqih & $\begin{array}{l}\text { Algérie } \\
\text { (opportu- } \\
\text { nité) }\end{array}$ & $\begin{array}{l}\text { Famille } \\
\text { (frères } \\
\text { sœurs) }\end{array}$ & $\begin{array}{l}\text { Ex employé } \\
\text { en Algérie. } \\
\text { Aujourd'hui } \\
\text { sans emploi }\end{array}$ \\
\hline Benaissa & $\mathrm{H}$ & 31 & $\begin{array}{c}\text { Tunisie } \\
1982\end{array}$ & $\begin{array}{c}\text { Depuis } \\
1986\end{array}$ & & $\begin{array}{l}\text { Schizo- } \\
\text { phrène } \\
\text { puis } \\
\text { bipolaire }\end{array}$ & $\begin{array}{l}\text { HO, } \\
\text { hospit } \\
\text { réité- } \\
\text { rées, } \\
\text { psy } \\
\text { privés }\end{array}$ & $\begin{array}{l}\text { Roqya, raki } \\
\text { Désensor- } \\
\text { celeuse } \\
\text { Taleb } \\
\text { Pharma- } \\
\text { copée } \\
\text { musulmane }\end{array}$ & $\begin{array}{c}\text { Tunisie } \\
\text { (opportu- } \\
\text { nité) } \\
\text { Tunisie } \\
\text { (opportu- } \\
\text { nité) } \\
\text { Toulouse } \\
\text { Toulouse }\end{array}$ & $\begin{array}{c}\text { Oncle, } \\
\text { parents } \\
\text { Incitation } \\
\text { amis } \\
\text { Incitation } \\
\text { ami } \\
\text { On lui } \\
\text { "en } \\
\text { parle» }\end{array}$ & Sans emploi \\
\hline Selma & $\mathrm{F}$ & 29 & $\begin{array}{c}\text { France } \\
1984\end{array}$ & & $\begin{array}{l}\text { Algé- } \\
\text { rienne }\end{array}$ & $\begin{array}{l}\text { Dépres- } \\
\text { sion }\end{array}$ & $\begin{array}{l}\text { Suivi } \\
\text { CMP }\end{array}$ & & & & $\begin{array}{l}\text { Étudiante/ } \\
\text { diplôme de } \\
\text { licence }\end{array}$ \\
\hline Issa & $\mathrm{H}$ & 53 & $\begin{array}{c}\text { Algérie } \\
1960\end{array}$ & $\begin{array}{c}\text { Depuis } \\
1965\end{array}$ & & $\begin{array}{l}\text { Toxico- } \\
\text { manie, } \\
\text { alcoo- } \\
\text { lisme }\end{array}$ & $\begin{array}{l}\mathrm{HO} \text {, } \\
\text { suivi } \\
\text { CMP }\end{array}$ & $\begin{array}{l}\text { (Religion) } \rightarrow \\
\text { rejet des } \\
\text { tradiprati- } \\
\text { ciens (islam } \\
\text { bid'aa) }\end{array}$ & & & Sans emploi \\
\hline Najib & $\mathrm{H}$ & 25 & $\begin{array}{c}\text { Maroc } \\
1988\end{array}$ & $\begin{array}{c}\text { Depuis } \\
2010\end{array}$ & & $\begin{array}{l}\text { Dépres- } \\
\text { sion }\end{array}$ & PASS & $\begin{array}{c}\text { Fqih } \\
\text { (Prières, } \\
\text { du'a) }\end{array}$ & $\begin{array}{l}\text { Avant } \\
\text { émigr. } \\
\text { Toulouse }\end{array}$ & Parents & Sans emploi \\
\hline Feza & $\mathrm{F}$ & 40 & $\begin{array}{c}\text { Tunisie } \\
1973\end{array}$ & $\begin{array}{c}\text { Depuis } \\
1993\end{array}$ & & Psychose & HDJ & $\begin{array}{l}\text { Imam : } \\
\text { Rokya } \\
\text { Fqih: } \\
\text { talisman, } \\
\text { hadra }\end{array}$ & $\begin{array}{c}\text { France } \\
\text { Tunisie } \\
\text { (opportu- } \\
\text { nité) }\end{array}$ & $\begin{array}{c}\text { Mari } \\
\text { Famille }\end{array}$ & \\
\hline Omar & $\mathrm{H}$ & 42 & $\begin{array}{c}\text { France } \\
1971\end{array}$ & & $\begin{array}{l}\text { Algé- } \\
\text { rienne }\end{array}$ & $\begin{array}{c}\text { Troubles } \\
\text { bipolaires, } \\
\text { délires } \\
\text { para- } \\
\text { noïaques }\end{array}$ & $\begin{array}{l}\mathrm{HO}, \\
\text { CMP }\end{array}$ & Talebs & France & Mère & \\
\hline Lounis & $\mathrm{H}$ & 31 & $\begin{array}{c}\text { France } \\
1982\end{array}$ & $\begin{array}{c}\text { Depuis } \\
1996\end{array}$ & & $\begin{array}{l}\text { Entend } \\
\text { des voix }\end{array}$ & $\begin{array}{l}\text { HJ, } \\
\text { hospit } \\
\text { réité- } \\
\text { rées }\end{array}$ & (Prières) & & & \\
\hline Ouardia & $F$ & 53 & $\begin{array}{c}\text { Maroc } \\
1960\end{array}$ & $\begin{array}{c}\text { Depuis } \\
1976\end{array}$ & & $\begin{array}{l}\text { Dépres- } \\
\text { sion chro- } \\
\text { nique }\end{array}$ & $\begin{array}{l}\text { Hospit } \\
\text { réité- } \\
\text { rées }\end{array}$ & $\begin{array}{c}\text { Rokya, } \\
\text { Rakih } \\
\text { Pèlerinage } \\
\text { marabout } \\
\text { (prières) }\end{array}$ & $\begin{array}{l}\text { Maroc } \\
\text { (opportu- } \\
\text { nité) }\end{array}$ & $\begin{array}{l}\text { Dont } \\
\text { oncle } \\
\text { Elle- } \\
\text { même }\end{array}$ & Sans emploi \\
\hline Saber & $\mathrm{H}$ & 20 & $\begin{array}{c}\text { France } \\
1993\end{array}$ & & $\begin{array}{l}\text { Maro- } \\
\text { caine }\end{array}$ & Psychose & $\begin{array}{l}\text { ITEP, } \\
\text { HJ, } \\
\text { CMP }\end{array}$ & & & & \\
\hline Salim & $\mathrm{H}$ & 31 & $\begin{array}{c}\text { France } \\
1982\end{array}$ & & $\begin{array}{l}\text { Tuni- } \\
\text { sienne }\end{array}$ & $\begin{array}{l}\text { Bipolaire, } \\
\text { bouffées } \\
\text { délirantes }\end{array}$ & $\begin{array}{l}\text { HO, } \\
\text { hospit } \\
\text { réité- } \\
\text { rées }\end{array}$ & (prières) & & & $\begin{array}{l}\text { Diplôme de } \\
\text { licence }\end{array}$ \\
\hline Farah & $\mathrm{H}$ & 35 & $\begin{array}{c}\text { France } \\
1978\end{array}$ & & $\begin{array}{l}\text { Algé- } \\
\text { rienne }\end{array}$ & Psychose & $\begin{array}{l}\text { Hospit, } \\
\text { HJ, } \\
\text { CATTP }\end{array}$ & (prières) & & & \\
\hline Rida & $\mathrm{H}$ & 25 & $\begin{array}{c}\text { France } \\
1988\end{array}$ & & $\begin{array}{l}\text { Maro- } \\
\text { caine }\end{array}$ & & Hôpital & Taleb & France & Parents & \\
\hline Aïcha & $\mathrm{F}$ & 39 & $\begin{array}{c}\text { France } \\
1976\end{array}$ & & $\begin{array}{l}\text { Maro- } \\
\text { caine }\end{array}$ & & & $\begin{array}{l}\text { Désensor- } \\
\text { celeuse }\end{array}$ & Maroc & Mère & \\
\hline
\end{tabular}


NB : nous avons signalé la religion entre parenthèses dans la colonne pluralisme médical.

C'est qu'elle n'est jamais présentée comme soin ou traitement, mais soulagement. CMP : Centre Médico-Psychologique; HO : Hospitalisation d'Office; PASS : Permanence d'Accès aux Soins de Santé; HJ : Hôpital de Jour; ITEP : Institut Thérapeutique Éducatif et Pédagogique; CATTP : Centre d'accueil thérapeutique à temps partiel.

* N'ayant pas accès aux dossiers médicaux ou au diagnostic du référent médical, les auteurs ont cherché à s'informer directement auprès des malades sur leurs pathologies.

À défaut de catégories nosographiques clairement identifiées, il a fallu parfois se contenter de manifestations cliniques telles qu'elles étaient rapportées par les malades ou observées par les auteurs. Cette démarche d'attribution d'une pathologie mentale a pu créer des difficultés notamment auprès des personnes les plus atteintes par la maladie ou les traitements - problèmes d'élocution, de compréhension, discours délirant, incapacité à savoir ce dont on souffre - posant en cela une limite à l'enquête de terrain.

** Si quelques données ont pu être recueillies sur l'emploi et le niveau d'études, les informations concernant cette catégorie restent partielles. Notons toutefois qu'en raison de leur état de santé, les informateurs.trices étaient dans leur grande majorité dans l'incapacité d'exercer un emploi ou d'étudier.

Source : Sicot et Touhami, 2014. 
réalisés soit dans ces structures soit à leur domicile ${ }^{7}$. Les patients interviewés avaient tous des droits ouverts qui leur permettaient d'accéder à la psychiatrie. Le tableau 1 présente quelques caractéristiques de ces patients - les prénoms sont fictifs afin de garantir leur anonymat - et de leurs recours. Par ailleurs, quatre psychiatres libéraux ont été interviewés à Rabat au Maroc. Comme on le verra, la recherche reste exploratoire et laisse ouvertes de nombreuses questions sur ce secteur traditionnel.

\section{Prégnance et diversité des recours traditionnels}

Six des quatorze patients interviewés pratiquent la prière en rapport avec leurs troubles mentaux. Outre le fait d'être un pilier de I'Islam, la prière est avancée, par les pratiquants, comme un appui thérapeutique. Les vocables employés mettent l'accent sur l'effet positif d'une pratique qui "soulage", "fait du bien", "fait plus que du bien" "réconforte". Dans le droit fil de ce rite, on peut également citer l'usage des $d u^{\prime} a$ - les invocations à l'adresse de Dieu souvent placées en clôture de la prière rituelle - qui prennent la forme de demandes directes à la divinité en vue d'obtenir soutien et guérison.

Ici, pas besoin d'être formé ou initié à la langue arabe, chacun compose avec son propre rapport à la connaissance de la langue du Prophète à l'exemple de Salim qui, malgré de profondes lacunes religieuses - de mère française, il n'a été que partiellement socialisé en milieu musulman - a découvert la prière auprès de certains de ses amis alors qu'il était hospitalisé en psychiatrie; prière qu'il reconnait pratiquer sans maitriser les bases élémentaires du rite. À I'inverse Ouardia, qui est née et a grandi au Maroc dans un milieu traditionaliste, entretient un nouveau rapport à la pratique religieuse depuis l'apparition de sa maladie : "Maintenant j'écoute beaucoup le Coran tous les jours [...] la religion ça compte [...]. La religion, ça m'aide beaucoup». Si la forme de la pratique rituelle varie ici, la visée, elle, reste commune. Le rapport direct et intime aux forces agissantes du sacré, la possibilité d'être acteur du soin et la quête de soins dans un cadre licite éclairent le recours à la prière qui délimite un élément saillant dans le champ des réponses au trouble psychique.

Pour les cas les plus bénins, les patients et les mères de famille mobilisent les savoirs de la médecine familiale, somme de connaissances vulgarisées tirées de différentes traditions médicales au Maghreb (Touhami, 2010). Cet ensemble composite se distingue en un registre prophylactique où il s'agit de prévenir le désordre qui, selon les interprétations culturelles du malheur, est associé à une agression surgissant de l'extérieur. Pour prévenir l'attaque de mauvais œil, étiologie qui peut être avancée dans les situations de troubles de I'humeur - dépression passagère, crises de pleurs chez l'enfant - on utilisera des pratiques basées sur le principe de contagion à l'instar des talismans coraniques portés à même la peau. Lorsque I'envoûtement est avéré, des rites curatifs sont utilisés pour soigner le malade. À cet escient, seront manipulés par les mères différents produits tirés de la

7 Afin d'obtenir la confiance des interviewés, les entretiens ont été menés parTouhami, ethnologue d'origine marocaine. Si la maitrise de savoirs culturels - connaissance de l'arabe dialectal, normes et codes culturels, contenus d'un espace therapeutico-religieux, etc. - a indéniablement favorisé le travail de collecte ethnographique, elle ne doit pas occulter l'ensemble des présupposés implicites sur le chercheur et les enquêtés. 
pharmacopée arabo-musulmane : pierre d'alun, sel, benjoin, encens, etc.

Certains commerçants proposent des réponses thérapeutiques traditionnelles aux maux qui accablent l'existence. C'est le cas des droguistes-herborises repérés sous les noms de attarines - les épiciers de quartiers - ou assabines - les marchands de simples, la barrière entre ces deux groupes de professionnels ayant tendance à se désagréger depuis quelques années au point qu'ils ne forment plus actuellement qu'un seul corps de métier (Bellakhdar, 1997, 70). Sollicités par les malades, ils proposeront à leurs clients les ressources tirées de la pharmacopée arabo-musulmane (ibid.).

En France, les commerçants de bazar orientaux prolongent leurs rôles et leurs fonctions en migration. À Toulouse, ils se sont établis dans plusieurs quartiers des grands ensembles en périphérie du noyau urbain. À l'instar des herboristes qui occupent une fonction de thérapeute populaire au Maghreb, ces commerçants peuvent poser des diagnostics ou prescrire des traitements à la demande leurs clients :

"Là-bas, au supermarché L.S [un bazar oriental], ils vendent de l'eau de la Mecque, l'eau de Zemzem'. C'est pour ça que je suis allé là-bas. Je lui ai dit [au commerçant] que je suis empoisonné. II me dit: "comment ça, empoisonné ?" Le mec m'a dit "ça ça vient de Médine, de la Mecque". II m'a dit: "tiens, le miel, tu mets ça dedans." "(Benaissa)

Les tradipraticiens sont aussi représentés par les clercs musulmans. Présents dans les pays de départ, mais proposant leurs services également en migration, le fqih - savant versé dans la jurisprudence - et le taleb dont la racine TLB désigne l'étudiant en sciences religieuses ont en commun d'avoir une formation de lettré dans le domaine des sciences islamiques. Au côté de fonctions comme la direction de la prière ou la lecture du Coran à I'occasion de cérémonies religieuses, ces spécialistes peuvent être les agents d'une activité thérapeutique qui se manifeste dans des compétences comme la confection de talismans et l'exorcisme.

Formé à la roqya, somme de savoirs thérapeutiques elle-même intégrée à la Médecine du Prophète, compilation de préconisations et de conseils en matière de santé tirés des hadiths et de la Sunna, le raqih consulté par trois des patients occupe une position particulière dans cette dernière catégorie. Sa pratique connait un regain d'intérêt en marge de la réislamisation des migrations issues des Suds. Initialement portée par la doctrine salafi qui prône un retour à la tradition des "pieux ancêtres" - les salaf, le raqih revendique une pratique conforme au modèle prophétique. Popularisée sur les sites d'hébergement de vidéos, accessible au public grâce à des traductions françaises et à des sites Internet spécialisés, cette doctrine est mobilisée pour traiter la possession djinnique, I'attaque de mauvais et la sorcellerie. Selon Cherak (2013: 297) qui a étudié cette pratique et ses évolutions en Égypte en Algérie et en France, "son expansion à l'échelle internationale témoigne de l'adaptation de représentations et de pratiques islamiques traditionnelles à la modernité et aux réalités socioculturelles locales".

8 Eau sacrée chargée de Baraka, on lui prête les mêmes vertus curatives que l'eau de Lourdes. Toutes deux sont d'ailleurs en vente dans des boutiques ou sur Internet. 
Seule Ouardia a eu recours aux marabouts dans notre échantillon. Le terme désigne ici les saints et leurs descendants. Leur pouvoir thaumaturgique résulte de leur baraka, force bienfaisante de nature divine que I'on retrouve dans le Coran, chez le Prophète et ses descendants ou dans des espaces sacralisés. À I'instar de ce que l'on peut observer en contexte catholique, il existe des saints spécialisés dans différentes maladies. Au Maroc, Sidi Mimoun au nord du pays et Sidi Ben Achir à Salé sont réputés pour leur pouvoir de guérison sur les troubles mentaux. Mais d'autres lieux peuvent être fréquentés, moins connus, mais proches du lieu de résidence de la famille.

" J'ai demandé à une cousine de ma mère de m'accompagner au wali [tombeau du saint]. C'est vraiment à côté, comme si tu allais au coin de la rue ici. J'y suis allée et j'ai invoqué Moul el Bergui.» (Mme Ouardia)

Enfin, Aïcha et Benaissa ont eu affaire à une désensorceleuse. Experte dans le traitement du sohr, elle n'en reste pas moins une figure marginale qui, de par son sexe et ses savoirs non indexés au Texte, reste exposée à des accusations de pratiques antireligieuses et antisociales qui l'amènent à exercer son art dans la discrétion.

La richesse de l'offre et la variété des recours ne doivent pas occulter leurs différences voire ce qui les oppose. Les tenants de la roqya se posent en s'opposant aux autres tradipraticiens musulmans - talebs, marabouts, voyantes dont ils critiquent les pratiques proches de l'associationnisme - le shirk - qui consiste à associer Dieu à autre chose qu'à lui-même (Touag, 2012). Des patients ont des recours multiples en fonction des opportunités et de leur trajectoire de malade quand $d$ 'autres établissent une distinction très nette entre ce qui est autorisé (halal) et ce qui est proscrit (haram). L'exigence de soin en conformité avec un modèle licite est présente dans les entretiens :

"Ça ne se fait pas, c'est haram d'agir de la sorte [...], il y a l'islam charia [orthodoxe, la voie droite] et l'islam bid'aa [innovations]. Je vois de mes yeux, quand ils font un talisman, les gens le vénèrent [le talisman].

- Ça, vous estimez que c'est pas bien? - Non.

- Pourquoi?

- Dans la religion, on ne doit pas aller voir le marabout."

Si le nombre de patients interviewés ne permet pas d'apporter de réponse définitive sur ce point, les recours traditionnels apparaissent tout de même comme des "bricolages culturels" qui s'inscrivent en partie dans le contexte du renouvellement de I'Islam en France. Ils peuvent relever de ce que Kepel (2012) nomme l'extension du domaine du halal, avec l'alimentation, le mariage (Collet et Santelli, 2012). Ils peuvent également éclairer les rapports très différents que les musulmans de France entretiennent à la religion. La prière de plusieurs des patients interviewés se réfère à un islam traditionnel, pratiqué par les membres de la famille restés au pays et encastré dans un mode de vie religieux. Par opposition, plusieurs jeunes interrogés (Salim, Farah, Issa, Lounis) prient, mais de manière récente, sans connaitre le Coran ni avoir d'autres pratiques religieuses.

Pour finir, il faut évoquer deux types de réponses à la maladie que n'ont pas évoqués les patients interviewés, mais qui reviennent régulièrement dans les 
propos de différents acteurs périphériques rencontrés lors des deux précédentes enquêtes : soignants, travailleurs sociaux, proches (sœur, parents). La première consiste à vivre sa maladie au pays, dans un contexte défini à la fois comme plus tolérant, mais aussi plus contenant, notamment avec la présence d'une famille élargie où peuvent se maintenir des formes de solidarité internes vis-à-vis des membres plus vulnérables. La seconde renvoie à la pratique du mariage endogame - systématiquement un homme si l'on s'en tient aux énoncés recueillis - avec la "cousine du bled». L'option du mariage traditionnel entre cousins, norme matrimoniale traditionnelle en contexte culturel maghrébin ${ }^{9}$ retrouve ici une nouvelle légitimité, l'union avec une parente socialisée au pays - plus docile et dévouée qu'en France d'après les témoignages consignés faisant alors office de soutien et support auprès du malade.

L'importance prise par le religieux dans le secteur traditionnel n'a rien d'original puisque de manière générale, la santé et les religions sont liées. D'un côté, tant l'anthropologie de la religion que l'anthropologie de la maladie montrent le pouvoir de guérison prêté aux pratiques religieuses, dont la prière. D'un autre côté, si l'on suit Laplantine (1982), I'entremêlement du religieux et du médical est une caractéristique essentielle des médecines qu'il nomme populaires. Un numéro des Archives de sciences sociales des religions montre la diversité, dans les sociétés contemporaines, des intrications de la religion et de soins psychiques, avec plusieurs articles qui relèvent son renouvellement dans le cadre des conceptions holistiques de la santé et de la maladie. "Religieux" et "psy" occupent les mêmes territoires, celui des définitions de I'humain, celui des affects qui peuvent déborder des corps, celui de la souffrance de l'âme ou de l'esprit et des soins à leur apporter, celui encore des règles morales, tout particulièrement en matière de sexualité et de relations familiales " (Champion, 2013 : 9). On pourrait ajouter qu'ils concourent tous les deux à fournir un sens au malheur.

Par ailleurs, ces recours traditionnels, s'ils tiennent leur spécificité des pays où ils trouvent leur origine et de la religion musulmane au sein de laquelle ils s'inscrivent en grande partie sont dans leur existence même banals. Le pluralisme médical et les recours traditionnels sont, dans le cas des troubles de la santé mentale en tout cas, fréquents. "Pour soigner leurs souffrances psychiques, les populations n'ont pas attendu que se développent des offres normées : depuis des siècles, elles consultent des praticiens dans le champ du religieux et de ses marges (guérisseurs, désenvoûteurs, exorcistes, etc.) pour des préoccupations relatives autant à l'âme qu'au corps [...]. Voir un psy n'est qu'une option parmi d'autres en santé mentale" (Kessler-Bilthauer et Evrard, $2018: 9$ ).

Ces recours traditionnels se font de manière pragmatique. Face à une maladie dont les causes sont mal connues et dont les évolutions sont incertaines, on sait que les individus recourent à tout ce qui pourrait leur apporter une aide, sans avoir besoin de construire une cohérence. Encore faut-il que ces aides soient disponibles. L'enquête montre que dans une grande ville française et, on le verra plus loin, en retournant parfois au bled, les recours cités sont disponibles dans leur diversité.

9 II existe une littérature fournie sur le sujet. Citons pour exemple les travaux de référence de Tillon (1966) ou de Lacoste Dujardin (1996). 


\section{Les articulations de la psychiatrie et des recours traditionnels}

On ne peut explorer des itinéraires thérapeutiques sans chercher à cerner comment s'ordonnent les différents recours. On sait que les personnes peuvent mobiliser de manière complémentaire divers types de soins ${ }^{10}$, la question qui est débattue étant dès lors de savoir comment ils s'articulent. À partir de I'analyse des itinéraires thérapeutiques des quatorze patients concernés dans notre enquête par le pluralisme, il n'est pas possible de dégager de règles générales relatives à l'articulation de la psychiatrie et des réponses traditionnelles. Ainsi les traitements psychiatriques peuvent-ils intervenir: 1. En priorité, avec la survenue de la maladie, particulièrement si une crise l'accompagne (Issa); 2 . En parallèle parce que le patient considère que son état relève de deux étiologies et donc de deux types de recours (Benaissa); car ils apportent leur bénéfice propre (Feza) ou encore parce que le traitement médicamenteux de la psychiatrie est nécessaire, mais insatisfaisant (Ouardia); 3 . Après que les recours traditionnels ont échoué à soulager ou à soigner (Omar, Amar).

Les soins traditionnels peuvent en effet être pratiqués en cas d'échec d'une réponse biomédicale, situation qui peut être illustrée par le récit de Rida, un jeune homme de vingt-cinq ans d'origine marocaine. Après un énième refus de stage en entreprise et sujet à des frayeurs qu'il ne s'explique pas, Rida a d'abord consulté à l'hôpital où il a pu bénéficier d'un scanner cérébral qui ne repérera rien d'anormal. Comme ses troubles persistent malgré un traitement médical, ses parents décident de consulter un taleb local qui pratique alors un exorcisme pour expulser avec succès les trois djinns - deux mâles et une femelle - qui tourmentent le jeune homme dont l'état s'est depuis amélioré, même s'il reste toujours précaire. Chez certains, comme Najib, la quête de soins pour atténuer les douleurs et les échecs successifs les amènent à utiliser toutes les ressources existantes, que ce soit en France ou au Maroc, à savoir d'abord les différents spécialistes biomédicaux, qu'ils exercent dans le secteur public ou privé, qu'ils pratiquent leur art dans le champ somatique ou psychique (psychologues, psychiatres), qu'ils opèrent ou qu'ils prescrivent des médicaments (antalgiques), mais aussi les différents tradipraticiens, les recours non conventionnels (hypnose), la prière, etc.

Pour les soignants de psychiatrie interrogés, les personnes feraient appel à eux après l'échec des recours traditionnels et l'aggravation de leur état. Ce cas de figure existe parmi d'autres situations. Ainsi Omar narre-t-il son itinéraire en évoquant d'abord des délires et des actes qu'il ne comprend pas, qui "ne lui correspondent pas". II dit en particulier : "Je faisais I'homosexuel». II interprètera ce qui lui arrive comme relevant d'une attaque de sorcellerie. Le recours au taleb participe de son passage à l'acte (meurtre) dans la mesure où, s'il est le premier à se considérer comme victime d'un sohr - l'agression sorcellaire - sa mère le confirmera dans cette idée ainsi que les différents taleb consultés :

10 Sur ce point s'accordent les travaux qui portent sur les médecins populaires, les recours non conventionnels, les soins traditionnels. 
"Vous l'avez tué pourquoi?

- Parce que je croyais que l'origine du sohr, c'était cette famille.

- Et vous avez tué celui que vous pensiez qu'il était le responsable.

- Voilà.

- Et vous, comment vous expliquez ce qui vous est arrivé ? Cette maladie?

- Moi au début, j'ai cru que c'était de la sorcellerie."

Omar sera hospitalisé d'office en psychiatrie puis en unité pour malades difficiles (UMD). Pour autant, la succession des recours ne relève pas d'un mécanisme simple, d'une conclusion logique tirée par le patient ou son entourage à la suite d'une expérience de soins. D'abord, la maladie du médecin (disease) n'est pas la maladie du patient (illness). Ensuite, l'échec et la réussite des recours sont des notions très relatives. La réussite de la psychiatrie est très diversement appréciée. La majorité des patients interrogés se plaint que la psychiatrie se réduise à la prescription de médicaments. Et les effets secondaires des médicaments sont soulignés par l'ensemble des personnes interrogées ${ }^{11}$. C'est même une des raisons pour poursuivre des soins traditionnels ou prier: ces pratiques viennent soulager à la fois de la maladie et des effets secondaires des médicaments.

"Ça aussi, c'est le réflexe de dire "c'est le traitement qui me rend fou. Donc j'arrête le traitement. Je vais voir le taleb" [...] Ça, on le sait après coup, par exemple, quand la personne va bien. " (Infirmier psychiatrique)

La "médecine de I'Islam» peut être considérée comme le traitement de fond quand la psychiatrie ne propose que des succédanés, des "médicaments pour dormir", qui vont aider pendant quelque temps, mais qui vont seulement "étouffer la maladie" sans s'attaquer aux causes. Le recours traditionnel s'explique donc en partie par les caractéristiques de la pratique biomédicale, son vécu, et par l'absence de sensibilité du psychiatre à la culture du patient, les difficultés de compréhension mutuelles.

Néanmoins, passer des soins traditionnels aux soins biomédicaux ne signifie pas qu'on abandonne les premiers ni que l'on cesse de "croire" à leur efficacité. Omar par exemple a prévu d'aller zohr (pratiquer le pèlerinage thérapeutique) sur un wali (tombeau de saint) en Algérie quelques mois après sa sortie de psychiatrie. L'hypothèse d'un enchaînement du recours traditionnel vers la psychiatrie au long d'un itinéraire thérapeutique repose sur l'idée que les patients croient en l'efficacité d'une thérapeutique et qu'ils peuvent être déçus par le constat de l'absence de résultats, c'est-à-dire qu'ils ont un raisonnement basé sur les preuves. En fait, les patients n'expriment pas une croyance sans distance critique. Certains disent même à propos de leur recours traditionnel : "ça se fait". D'autres arguments sont du type "ça ne peut pas faire de mal" et "on ne sait jamais". Et puis pour les acteurs, un ensemble de raisons peut expliquer que "ça n'a pas marché". Enfin, pour les patients tombés malades à l'étranger, le recours traditionnel en première instance s'explique autant par leur disponibilité, leur faible coût et l'absence d'offre en psychiatrie que par la croyance ou la culture. Les patients et leurs proches ne sont pas ancrés dans une culture d'origine qui expliquerait leur recours pour la bonne raison

11 Ceci n'est en rien spécifique aux migrants (Palazzolo et al., 2008). 


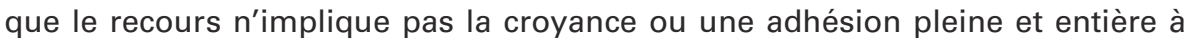
des croyances ${ }^{12}$, une étiologie, et que les anthropologues ont pu montrer que les représentations (de la maladie, de ses causes) entretenaient des relations complexes, non systématiques avec l'action thérapeutique mise en œuvre ${ }^{13}$.

Les différents recours sont mis en œuvre tout au long des itinéraires thérapeutiques, et manifestent l' "éclectisme pragmatique» des patients (Monteillet, 2005 : 189). Cette situation, la plus fréquente, privilégie le principe de complémentarité à la concurrence, les différentes réponses thérapeutiques utilisées se justifiant au regard de "la recherche d'action, d'efficacité, dans le désir de transformer positivement un état" (Benoist, $1996: 496$ ).

\section{Les itinéraires thérapeutiques des migrants originaires du Maghreb se distinguent-ils de ceux de leurs héritiers?}

Nous avons rappelé dans la première partie de cet article la nécessité de prendre en compte tous les déterminants sociaux et économiques de vie en France, la trajectoire migratoire, la situation du migrant avant son émigration, les droits à l'accès aux institutions pour éviter d'expliquer les pratiques de soins des migrants par l'appartenance à un groupe culturel homogène et une culture d'origine (Fassin, 2000a ; Wang, 2016). Si nos entretiens avec les patients comportent des informations sur ces déterminants, ils ne sont toutefois pas suffisamment nombreux et probants pour expliquer les recours au secteur traditionnel. Par contre, les analyses nous ont amené à explorer une autre piste, non exclusive, celle du changement culturel.

Le recours au secteur traditionnel apparaît fréquent à la fois chez les migrants du Maghreb et chez leurs héritiers. Pour autant, ne peut-on pas supposer que les premiers soient plus concernés que les seconds? Que le temps passé en France, le lieu de la socialisation primaire influent fortement sur les chances de recourir au fqih ou au désencorceleur? Bref, que l'acculturation estomperait des références venues d'ailleurs? Une telle hypothèse repose sur l'idée d'un processus temporel linéaire de prise de distance vis-à-vis des éléments culturels issus du pays d'origine. Or nous savons que le temps est loin d'être le seul critère permettant de comprendre la dynamique des recompositions culturelles. Certains auteurs ont montré en particulier le rôle dans la transmission de la mémoire, des valeurs et des manières de faire des orientations politiques nationales en matière de citoyenneté (Streiff-Fénart, 2006); du projet migratoire; de l'entre-soi créé par la reléga-

12 En écho à cette idée, on citera cette psychiatre qui met en rapport l'attachement à une "croyance" avec la situation sociale vécue en France: "Souvent ces jeunes de la deuxième génération se réapproprient des éléments de culture et/ou religieuse parfois, mais parfois avec une construction solide parfois non, mais une volonté d'appartenance indéniablement. Après cette volonté d'appartenance, il y a différents déterminants. Parfois c'est une volonté d'appartenance positive et parfois c'est pour s'opposer à autre chose, au sentiment de rejet que peut leur renvoyer la société française. Et du coup ils renvoient cette appartenance, mais de manière superficielle. Mais je n'ai pas à juger, tant que leur truc fonctionne... ". Dans une tout autre perspective : sur la question de la différence à opérer entre croyance collective et degré d'adhésion personnel, voir Sakoyan (2008) et Rechtman (2000).

13 Voir par exemple le numéro thématique de Sciences sociales et santé de 1985. 
tion dans des quartiers périphériques (Khosrokhavar, 1997); d'autres ont souligné l'importance de la revendication identitaire (Poutignat et Streiff-Fénart, 1995), étudié la diversité des modalités de transmission culturelle au sein des familles, les liens entretenus avec le pays d'origine via le commerce ou l'entrepreneuriat ${ }^{14}$, les liens familiaux (Razy et Baby-Collin, 2011), la réception des médias arabes (Hargreaves et Mattelart, 2012) et les vacances au bled (Bidet et Wagner, 2012).

Existe-t-il une distinction entre patients migrants et patients héritiers? II est à noter que cette dichotomie n'est pas retenue par les soignants lorsqu'ils évoquent le pluralisme médical de patients originaires du Maghreb. D'après les données recueillies, la nationalité ne semble pas constituer non plus une catégorie discriminante. Comme le présente le tableau 1, le fait de recourir ou non au secteur traditionnel n'est pas lié au lieu de naissance. Parmi les quatorze patients interviewés, trois de ceux qui sont nés en France ont bénéficié des services d'un taleb ou d'une désensorceleuse. Si c'est le cas de cinq des six informateur.trice-s né.e.s à l'étranger, précisons toutefois que deux sont arrivés en France durant la petite enfance et une à l'adolescence. Par conséquent, c'est moins le temps passé en France que les savoirs acquis pendant la socialisation primaire et entretenus tout au long de la vie qui semblent expliquer la prégnance des savoirs et des pratiques traditionnels. Trois types de situations - regroupant onze des quatorze interviewés - sont relativement clairs au sens où ils permettent effectivement de rapprocher niveau d'acculturation et rapport aux recours traditionnels.

Dans le premier groupe, les individus ont des recours congruents avec des savoirs étiologiques : la représentation étiologique commande le traitement. La maladie résulte de l'action d'un agent étranger, ici un sorcier ou un génie (modèle exogène), et l'action thérapeutique consiste à soustraire l'élément fautif $^{15}$ (modèle soustractif, voir Laplantine, 1986). Le fait d'être possédé, d'être "aïen " (victime mauvais œil) va de soi. On parle d'avoir la niya (la croyance). Le délire est codé culturellement :

" Les voix me parlent de l'autre monde en Islam. J'étais avec des anges. Il y avait des anges qui vivaient avec moi dans l'immeuble où j'étais à Nancy.

- Vous les voyiez? Vous discutiez avec eux?

- Je les voyais. Je leur demandai s'ils voulaient de la pizza.

II me disait qu'ils n'en mangeaient pas.

- Ces personnes, ce sont des anges ou des génies (djinns)?

- Des anges. Les génies m'ont malmené au pays."

Les soins traditionnels sont évoqués spontanément et font manifestement partie de l'univers mental quotidien, avec de nombreuses références au pays d'origine : comment on y vit, comment on obtient de l'aide en cas de malheur. Les recours au fqih, aux désencorceleurs, les pratiques de protection sont des évidences.

14 Voir notamment les travaux de Tarrius (1993).

15 "Quand tu te lèves le matin, avec un verre d'eau de la Mecque, mélangé à l'eau normale, haba saouda, tu manges 7 dattes et tu bois. - Vous l'avez fait ça? - Je I'ai fait. J'ai vomi des trucs bizarres accrochés aux dattes. Ça te fait tout gerber ou ça te fait aller aux toilettes [...]. La haba saouda, quand tu la prends, elle va dans le sang, ça fait mal au cour. Tu sens parfois des sueurs froides. Ça évacue [...]. Ça enlève tout." (Benaissa). La haba saouda, littéralement la graine noire, désigne la graine de nigelle abondamment utilisée dans la pharmacopée arabo-musulmane. 
L'expérience migratoire des individus concernés est extrêmement hétérogène puisque trois sont nés en France (Omar, Rida, Aïcha), deux sont arrivés très jeune (Benaissa et Ouardia), mais ont passé de nombreuses années en France, une est arrivée à l'âge de vingt ans, mais est installée depuis vingt ans (Feza). Le cadre cognitif dans lequel s'inscrivent les recours ne s'explique pas (seulement) par le temps passé au Maghreb, mais aussi par l'importance prise par les liens communautaires au sens deTonnies c'est-à-dire fondés sur l'affectivité et l'esprit de groupes restreints, unis par des relations directes et étroites, que ce soit en raison de liens de sang (la famille élargie), une référence fréquente aux proches du voisinage ou à ceux qui sont restés au bled. Les cinq individus concernés font des aller-retour fréquents au bled et font état d'une forte densité de leurs relations sociales familiales en France et au Maghreb.

Dans le second groupe, on trouve trois jeunes Français nés en France résidant dans des quartiers prioritaires de la politique de la ville (Lounis, Saber, Farah). Ils n'expriment aucune croyance en une étiologie exogène ou une malédiction. La maladie résulte d'une cause matérielle ou humaine accidentelle. Leur recours hors psychiatrie se limite à la prière. Si deux se disent croyants, ils ne connaissent pas la religion islamique, ne savent pas non plus comment prier. Ils ne se sont mis à prier qu'avec l'irruption de la maladie. Les vertus qu'ils prêtent à la prière sont simples : elle soulage. Sur les quartiers de relégation, la prière est d'ailleurs parée de cette vertu générale et il n'est pas rare qu'on s'y livre pour soulager l'ensemble de maux causés par la vie difficile : I'absence de travail, des ennuis avec la famille, la justice, etc. ${ }^{16}$. Leur culture semble être celle qu'ont décrite les auteurs qui ont travaillé sur les jeunes des banlieues et qu'ils nomment la "culture des rues" avec ses codes, ses rites et ses langages (Lepoutre, 1997; Avenel, 2009). Les pairs constituent la quasi-totalité des relations sociales citées.

" J'ai toujours habité là. J'ai grandi là. Je connais tout le monde. Je connais la violence. Je connais les filles. J'ai connu la prison. Je connais les boîtes. Je connais les voitures. J'ai connu les motos. " (Farah)

La cité constitue la référence des sociabilités et des expériences sociales. Si I'on a pu aller au bled pendant l'enfance, on n'y a pas gardé d'attaches.

"Vous étiez petit... Vous n'y êtes pas retournés depuis? - Non. J'ai dû y aller une ou deux fois à peu près. Ça m'avait plu. C'était bien, mais c'est un peu dans l'oubli quoi. - Comment?

- Dans ma mémoire c'est un peu à zéro. - Vous n'avez plus de souvenirs? - À part les animaux, le couscous avec les boules. Je me rappelle de ça, la télévision aussi. Les chaînes marocaines. " (Lounis)

Selma et Salim représentent une autre configuration. Ils n'ont aucun recours hors la biomédecine et la psychologie. Ils ont quasiment le même âge, sont nés en France et ont grandi dans des quartiers mixtes, au sein de familles de la petite

16 Un éducateur à un jeune, lors d'une activité sportive : "La prière, la prière. Ça permet d'évacuer, de te vider la tête. Tous les soucis; tu oublies. La salle c'est bien, mais tu vas voir, la prière, ça fait du bien" (note d'observation). 
classe moyenne qu'ils décrivent comme laïques. Ils ont fait des études et ont tous deux obtenu une licence. Soit ils ne se réfèrent jamais dans l'entretien à la culture du pays d'origine de leurs parents, soit ils marquent une nette distance vis-à-vis de celle-ci. Selma exprime une grande distance avec la "culture du bled ", selon ses propres termes, et pas seulement quant aux soins traditionnels dont elle dit : "je $n^{\prime} y$ crois pas, c'est du pipeau ». Elle mêle ensuite la croyance au mauvais œil avec d'autres références, dans une sorte de pot-pourri :

" Je crois au mauvais œil parce que je crois aux mauvaises ondes en fait. Je crois que s'il y a plusieurs personnes qui me veulent du mal. Je crois au truc des Asiatiques, le Ying et le Yang. Ça fait du bien et tout. Si on fait du mal, ça se répercute."

En tout cas, elle ne s'est jamais protégée contre le mauvais œil. Durant son enfance, elle a très peu été au bled: " je n'étais pas trop tenté sur le bled. Moi j'étais la vraie francisée ". À de nombreuses reprises, elle se présente comme française et se distingue des "Arabes " : son propre mari, qui a "la mentalité des hommes du bled ", ses beaux-parents qui ont "la culture du mensonge " et même sa famille (" Mon grand frère battait mon petit frère quand il faisait des bêtises, c'est typique chez les familles maghrébines "). D'un autre côté, c'est la seule interviewée à être satisfaite de sa relation avec les psys (psychologues et psychiatres) ce qu'elle explique en disant : " elles sont d'origine maghrébine. Elles connaissent les problématiques liées à la culture maghrébine. C'est vachement plus efficace ".

Les recours d'Issa, de Najib et de Amar ne semblent pas pouvoir être rapprochés d'un quelconque niveau d'acculturation. Né en Algérie, arrivé en France à l'âge de cinq ans, $\mathrm{M}$. Issa est celui qui incarne le mieux le fait de vivre ici et là-bas. II dit d'ailleurs : " Je vais et je viens. La navette voilà [...]. J'ai grandi là-bas jusqu'à l'âge de six ans. Après pour les vacances d'été, on redescendait en Algérie». Cas rare, sa mère née en France fait partie des colons partis s'installer en Algérie, dans son cas à I'âge de dix-neuf ans. Elle s'y est mariée avec un Kabyle. Ils sont rentrés en France au moment de I'Indépendance, mais la plupart des membres de la famille, dont une partie de la fratrie d'lssa, est restée en Algérie. Sa mère, qui adopte la religion de son époux, parle arabe fait le ramadan et la prière. Comme le chercheur lui fait remarquer qu'il parle avec un fort accent algérien, il répond que : "C'est à force. On a toujours discuté à la maison sur l'Algérie. En algérien. On ne parle pas français avec mon père. Toujours en algérien même avec ma mère". Très pratiquant, il prie plusieurs fois par jour. S'il refuse d'aller voir un fqih ou de faire un pèlerinage, s'il rejette les recours traditionnels c'est pour deux raisons. D'abord parce que c'est haram, que cela ne représente pas la "voie droite». Ensuite parce que "ça ne vaut rien du tout. C'est juste pour le fric, du bizness". La critique qu'il fait des recours traditionnels est celle que l'on retrouve fréquemment dans les médias au Maghreb, dans les classes moyennes et supérieures : ce sont des recours traditionnels qui pour la plupart ont été dévoyés par des charlatans, des escrocs, qui donnent lieu à des abus financiers ou des maltraitances.

Quant à Najib, son cas a été évoqué plus haut : il représente de manière archétypale le patient désespéré qui recourt à tout ce qu'il trouve pour soulager sa souffrance. Né au Maroc et arrivé en France depuis seulement trois ans à l'âge de vingt-deux ans, il est issu de la petite classe moyenne d'une grande ville, il 
a commencé par consulter tout un ensemble de représentants de la biomédecine. Puis, en désespoir de cause, parce que les ressources étaient disponibles et à l'initiative de ses parents, il a fini par consulter un fqih. II émigre en France pour trouver une solution à ses souffrances où il consulte à nouveau plusieurs médecins : "J'ai juste tenté ma chance pour trouver une explication à mon problème de santé". Najib a toujours prié, au Maroc comme en France, mais la prière a pris un autre sens avec l'irruption de la maladie.

Amar est arrivé en France depuis peu après avoir passé l'essentiel de sa vie en Algérie, il était employé dans l'administration et a émigré suite, dit-il, à des menaces de mort. II a d'abord consulté un fqih en Algérie après son départ, à I'occasion d'un séjour forcé consécutif à une expulsion du territoire français. Le contexte social, la disponibilité, l'opportunité l'amènent à cette consultation, mais sans qu'il paraisse y adhérer beaucoup :

" J'avais vu un fqih, II m'avait donné un traitement, ça va. II faut avoir de la niya [la croyance] pour que ça marche. Ça ne marche qu'avec ça. On m'a envoyé vers lui. - Qui ça, la famille? - Oui la famille."

Pour autant, Amar n'est pas sceptique de manière générale vis-à-vis des étiologies exogènes ou de la possibilité d'une possession. S'il n'a pas été consulter un imam, c'est qu'il identifie l'origine psychosociale de ses souffrances: ses relations avec son entourage, son ex-femme : "Je ne suis pas possédé. Ce sont des problèmes qui me stressent». S'il a commencé à consulter un psychiatre en Algérie, il poursuit les consultations en France et désormais c'est au psychiatre français qu'il accorde sa confiance dans la mesure où le traitement le soulage :

"Quand vous rentrez en Algérie, vous allez le voir [le premier psychiatre consulté]? - Non. Je viens avec mon propre traitement. Il y a des médicaments que je trouve là-bas et d'autres sont absents.

- Vous n'allez pas voir de médecins quand vous rentrez? - Non. Il faut prendre rendez-vous et tout. Je pars avec mon traitement. Je n'ai aucune raison d'aller le voir."

Amar puise dans les ressources de soins disponibles, ici et là-bas. Il illustre le pragmatisme qui mène à poursuivre un type de soins parce qu'il semble le plus efficace pour réduire la souffrance. On peut croire à la possession sans recourir au secteur traditionnel de la même manière qu'on a vu qu'on pouvait y recourir sans pour autant croire.

Ces différents cas montrent qu'on ne peut établir un lien simple entre recours pluriel, degré d'acculturation et temps passé dans le pays d'accueil ni entre itinéraire thérapeutique et expérience migratoire. Certes ces dimensions ne sont pas sans expliquer en partie le choix des recours et le pluralisme. Mais apparaissent tout aussi important le rapport entretenu avec des références culturelles héritées via l'inscription dans des réseaux de sociabilité, qui peut être facilités par le fait de résider dans des quartiers de relégation, avec une présence forte d'habitants issus des mêmes pays, les aller-retour au bled, le milieu social d'origine ou le niveau de scolarisation atteint $c^{\prime}$ est-à-dire la trajectoire sociale et l'efficacité du traitement. 


\section{Se soigner ici et là-bas}

De quel côté de la Méditerranée les migrants et leurs héritiers cherchent-ils les tradipraticiens, les herboristes et les imams? Tous les cas de figure sont possibles : en France ou au Maghreb, en France et au Maghreb. Les soignants de psychiatrie interrogés savent que leurs patients originaires du Maghreb sont nombreux à se rendre au pays pour y recevoir des soins. La pratique n'apparaît donc pas marginale. Pour autant, ils ne savent pas ce que font leurs patients là-bas.

La disponibilité de l'offre constitue un déterminant essentiel dans le recours. Les spécialistes sont-ils présents et aisément accessibles en France, dans les villes, mais aussi en zones rurales? L'imam évidemment, mais aussi le fqih ou le taleb à l'exemple de ce professionnel rencontré dans une grande ville et qui se vante que "le téléphone n'arrête pas de sonner chez lui». L'épicerie, le supermarché du quartier fournissent les produits à ingérer, les herbes à faire brûler. Ce qui n'empêche que l'on ramène également des produits de ses séjours au bled, qu'ils circulent du Maghreb vers la France.

En France, les spécialistes sont connus et repérés, ont leur réputation qui circule par le biais du bouche-à-oreille : "On me l'a conseillé/Mes voisins m'en ont parlé». La proximité et la facilité de l'accès sont encore plus frappantes encore au Maghreb. C'est alors littéralement au coin de la rue que I'on peut trouver le spécialiste et les propos soulignent la massivité des recours, comme si tout le monde était concerné : "les gens y vont". Pour autant, tout n'est pas possible en France. Le retour au bled permet le pèlerinage sur le tombeau du saint. II rend également possible la visite chez ces passeurs de monde que sont les membres des confréries maraboutiques - Gnawas, Aissaouas - socialement reconnus dans le traitement de la possession djinnique (Chlyeh, 1997).

Pourquoi recourir ici ou là-bas? La disponibilité de l'offre rend possible le recours là où on tombe malade. Ainsi Ouardia dit-elle être tombée malade lors du retour au Maroc effectué pour enterrer sa mère. "J'ai fait des crises. Des personnes sont venues lire le Coran pour me soigner et tout [...]. Le soir vers six heures un jeune cousin m'a amené de l'eau de ZemZem». Plus tard, elle se rendra sur le tombeau du wali. M. Amar "n'allait pas bien» en Algérie, avant son émigration vers la France. De lui-même, il a été consulter un psychiatre. Parallèlement, la famille l'a envoyé voir le fqih. C'est encore le cas de Najib. Les autres personnes interrogées qui sont tombées malades en France ont recouru (d'abord) en France.

"Les fqih vous les avez vus en Tunisie ou ici?

- En Tunisie et ici. Parce que ma première maladie c'était ici.» (Feza)

D'autres arguments jouent en faveur du "là-bas», comme l'idée qui veut que puiser à l'origine soit plus efficace qu'en exil. Certains critiquent les tradipraticiens établis en France au motif qu'ils seraient moins compétents ou qu'ils ne disposeraient pas des bons produits. Une pratique que confirme une psychologue d'origine algérienne exerçant dans une PASS psychiatrie : "j'ai l'impression qu'il y a quand même cette idée que les marabouts d'ici, c'est pas pareil 


\section{[...]. Ils sont moins forts ${ }^{17}$.}

Le séjour au bled - dans le cadre des aller-retour réguliers, entre ici et là-bas, lors de vacances, de fêtes ou parce qu'on vit à la fois ici et là-bas, dans la maison que I'on a construite en Algérie et dans l'appartement où I'on réside en France (multirésidence) - peut être l'occasion d'aller voir un tradipraticien :

"Je suis allé au Maroc en août dernier.

- Chez des amis?

- Oui. Il nous a ramené quelqu'un du marché, une belle femme, vieille, mais belle, peau blanche, yeux bleus. Elle m'a fait boire de l'eau qu'elle fait bouillir avec de la réglisse et à la mosquée ils m'ont dit, "Ça, c'est pour t'enlever la sorcellerie et le mauvais œil". - C'était juste en vacances?

- En vacances, mais j'en ai profité.." (Benaissa)

Enfin, la famille peut se saisir d'un séjour au pays pour amener un de ses membres consulter un spécialiste. C'est le cas d'Aïcha, jeune Française d'origine marocaine d'une vingtaine d'années :

"Tout ça, c'est ma mère. Elle croyait qu'on m'avait jeté un sort pour pas que je me marie [...]. C'était pas facile pour moi à l'époque niveau boulot, le moral tout ça. Alors pour elle, c'était clair [...]. Quand on est retourné l'été au bled, elle m'a emmené voir une "seharra" [désensorceleuse]."

Lorsque Nathan (1994) a théorisé l'impérieuse nécessité du retour au pays pour y accomplir les rituels réparateurs et se faire soigner par la communauté et les spécialistes du pays d'origine, aux critiques de son culturalisme sont venues $s^{\prime}$ ajouter $d^{\prime}$ autres propositions thérapeutiques ${ }^{18}$. Notre enquête rappelle que les malades construisent aussi leurs itinéraires thérapeutiques hors des prescriptions des médecins et que le "retour thérapeutique" peut fréquemment être activé.

Cette capacité à mobiliser des ressources ici et là-bas se manifeste particulièrement dans la pratique qui consiste à aller consulter des psychiatres au Maghreb. La partie de l'enquête exploratoire menée auprès de psychiatres marocains à Rabat a ainsi permis de constater que des émigrés ou leurs enfants consultaient des psychiatres libéraux ${ }^{19}$ lors de leurs séjours au Maroc. Concentrés sur les grandes artères de la ville européenne - I'Agdal - qui fait face à la médina, l'ancien noyau urbain, les psychiatres rencontrés affirment accueillir régulièrement cette population dans leur consultation. C'est principalement le cas l'été où, d'après ces informateurs qui ont tous étudié la psychiatrie en France, leur file active est constituée de nombreux «RME» - abréviation

17 Là encore, I'idée n'est pas spécifique à I'Islam. À propos de Lourdes, Cogat-Droulez (2001) écrit : "Une des raisons principales de ces pérégrinations est la conviction que des prières et d'autres rituels religieux sont actifs dans des lieux où se sont manifestés, d'une manière ou d'une autre, le "Créateur", un saint ou une divinité ".

18 II est difficile de citer dans le cadre de cet article toutes les psychiatries transculturelles et les positionnements des uns et des autres vis-à-vis des propositions de Nathan (1994). Voir par exemple les travaux de Moro et Baubet (2013) ou Douville (2006).

19 Interrogés sur cette question, des psychiatres hospitaliers n'ont pas fait état de la même pratique. Par contre, il leur arrive d'hospitaliser en situation de crise des émigrés d'Europe de passage au pays. 
administrative pour résidents marocains à l'étranger - issus de différents pays d'Europe : France, mais également Italie, Espagne, Allemagne. La raison de ces consultations tient selon eux à leur double compétence, biomédicale et experte en étiologies et thérapeutiques traditionnelles. Non qu'ils pratiquent l'un et l'autre, mais ils savent entendre les interprétations du mal, y être sensibles - "Ils viennent nous voir, car ils se sentent mieux compris.", "Ils se sentent écoutés" - et proposent un traitement dans lequel l'articulation des systèmes culturels a été élaborée ${ }^{20}$.

Le fait que des malades puissent consulter des psychiatres et des spécialistes non professionnels ici et/ou là-bas invalide les dichotomies entre l'ici, la France qui représenterait la modernité versus là-bas, le Maghreb où l'on irait retrouver la tradition, accomplir les rituels ${ }^{21}$. Les recours aux secteurs traditionnel et professionnel ici et là-bas représentent une autre facette des pratiques transnationales des migrants et de leurs héritiers, à côté des activités commerciales et entrepreneuriales des migrants désormais bien étudiées. Mais en s'inscrivant uniquement entre la France et le pays d'origine, ils témoignent moins d'une compétence circulatoire (Tarrius, 1993) que de l'importance de l'ancrage dans les deux pays. La notion de "transnational " est l'objet de définitions multiples (Ma Mung et al., 1998) et renvoie tantôt à des réseaux multipolaires de migrants, tantôt au paradigme des relations bipolaires entre société d'émigration et société d'accueil (Sakoyan et al., 2011). Dans notre enquête, le transnational en question relève bien des liens forts entretenus par les migrants et leurs héritiers avec leur pays d'origine. Ils doivent moins à la globalisation et à la marchandisation de la santé qu'aux relations que les immigrés et leurs descendants gardent avec un pays d'émigration, les soins étant un des domaines où se concrétise cet attachement.

Ce ne sont pas uniquement les Maghrébins de France qui sont concernés par cette mobilité thérapeutique. En effet, Le Gall et Montgoméry (2009 : 4) ont observé les mêmes recours chez les Maghrébins du Québec : "L'analyse des itinéraires thérapeutiques des personnes interviewées met en évidence une conjugaison systématique et récurrente des usages de la médecine biomédicale et des "aides informelles", mais également un aller-retour entre pratiques locales et pratiques transnationales". II est même probable que d'autres migrants sont concernés. La littérature sur les mobilités thérapeutiques s'est particulièrement développée ces dernières années et a mis en exergue toute la diversité des circulations, au-delà du seul tourisme médical ${ }^{22}$. Pourtant, cette figure de la mobilité que représente le retour au pays reste extrêmement peu explorée au profit de l'étude de celles qui impliquent des individus qui vont chercher des soins hors de leur pays de naissance. "A substantial part of medical travel is of diaspora patients returning 'home' to familiar (and usually cheaper) circumstances. Like border crossers, little has been written on such travellers, because they are of limited significance to the industry, not easy to distinguish and even harder

20 Voir en particulier les travaux du psychiatre Bennani (1996) rencontré à Rabat.

21 Le recours aux tradipraticiens au Maghreb ne doit pas être réduit à une pratique culturelle (croyances et autres). Il s'explique aussi très largement par l'absence d'offre de soins biomédicaux. Pour l'Afrique plus globalement, on peut consulter Burns et Tomita (2015).

22 On parle désormais de transnational health care practices (Connell, 2015). 
to document. Yet the return migration of Indians effectively instigated medical tourism in India. Many patients travel home to familiar cultural and linguistic circumstances, partly because they cannot understand or afford local health care" (Connell, 2015 : 399). Si des motifs économiques peuvent expliquer de telles mobilités (Stan, 2015), nous faisons I'hypothèse avec Connell et à partir de nos données, que les migrants et leurs héritiers peuvent également trouver dans ces recours/retours au pays une confiance et une familiarité culturelle soignants/ soignés qu'ils ne trouvent pas dans leur pays d'installation ${ }^{23}$.

\section{Conclusion}

Étudier intégralement les itinéraires thérapeutiques de patients atteints de troubles mentaux nécessite de ne pas les considérer comme la résultante des seules représentations culturelles du corps, de l'esprit et de la maladie. Doivent être pris en compte également des conditions de vie et l'accès au système de santé dans ses différentes dimensions : la connaissance de ce système, le coût, les droits.

On doit tenir compte en outre du fait que tous les malades, migrants ou pas, ont la possibilité de se tourner vers un marché des soins de santé mentale vaste et pluriel. Kleinman a proposé une théorie de systèmes de soins qui permet d'ordonner d'une certaine manière cette offre. L'enquête a montré que des patients pouvaient chercher de l'aide du côté des secteurs biomédicaux et traditionnels, cette dernière catégorie comprenant aussi le religieux dans ses variations orthodoxes et hétérodoxes. En se centrant sur un seul secteur, il est alors difficile de reconstituer la totalité des itinéraires thérapeutiques. La recherche était moins focalisée sur la question de leur articulation - complémentarité, concurrence, succession - que sur la volonté de présenter une première exploration du secteur traditionnel auquel recourent les migrants maghrébins en France. Néanmoins, une thèse est discutée en anthropologie médicale, celle qui expliquerait le recours à des alternatives à la biomédecine par la quête de sens à la question du "pourquoi moi?"; question à laquelle les médecins ne répondraient pas. Or la recherche de sens ne nous paraît pas déterminante pour expliquer le recours à des alternatives par les patients enquêtés. Une partie seulement d'entre eux exprime une insatisfaction vis-à-vis de la psychiatrie et celle-ci concerne plutôt d'une part la réduction des soins à la prescription médicamenteuse et, d'autre part, l'absence de sensibilité culturelle, le sentiment de n'être pas compris dans sa manière de voir le monde et de s'y situer.

Aller plus avant dans l'étude du secteur traditionnel nécessiterait d'interroger les spécialistes concernés - fqih, imams, herboristes - au mieux d'observer leurs pratiques, les rituels auxquels ils se livrent, les traitements qu'ils délivrent. $C^{\prime}$ est ainsi que l'on pourrait savoir en particulier comment leurs savoirs s'implantent en France, ce qu'ils puisent à d'autres traditions et ressources, dans

23 Voir ainsi l'article de Green (2006 : 1506) qui va dans notre sens : "The health pathways of our participants show that Chinese migrants maintain a strong identification with their culture of origin ". On trouve également dans cet article de nombreux points communs sur les usages du pluralisme médical (pragmatisme, distance à la "croyance", etc.) de migrants résidants dans un pays où la biomédecine est très largement dominante. 
quelle mesure et sous quelle forme la "tradition" revendiquée se renouvelle et se réinvente dans un frottement constant avec d'autres références.

Le rapport que chacun entretient avec la culture au sein de laquelle il a été socialisé dépend d'un ensemble de conditions sociales, économiques et, pour les migrants, d'une trajectoire migratoire. Nous avons plus particulièrement ici tenté d'examiner le lien entre les recours au secteur traditionnel et les trajectoires migratoires, les relations entretenues avec le pays d'origine, le niveau d'acculturation, les réseaux de sociabilité. Ceux-ci semblent avoir effectivement un lien même si la disponibilité des ressources relevant du secteur traditionnel et le pragmatisme constituent des éléments déterminants.

Notre première exploration d'une facette du pluralisme a également laissé de côté la question de la recomposition culturelle ou plutôt, comme nous y incite Hammouche, la question des compositions. Car la notion de recomposition, «malgré les précautions qui l'accompagnent véhicule l'idée de plusieurs ensembles "fixés" dans leurs différences. Comme si "culture" s'entendait dans un temps statique et derrière une clôture spatiale que le métissage remettrait en question [...]. II y a constamment un processus de composition qui, selon des rythmes différents dans le temps, apparaît plus ou moins manifestement " (2007 : 202). La difficulté consiste à identifier comment et ce que les individus composent, ce qu'ils empruntent, reçoivent de la transmission, trouvent dans le pays d'accueil, d'autant plus si tous les éléments en cause ne cessent d'évoluer.

Dans notre enquête, les compositions ne tiennent pas seulement aux modalités et aux conditions de la transmission. Elles se nourrissent de la mobilité des acteurs qui recourent ici et là-bas, en rapportant du pays d'origine des produits, mais aussi des idées, des manières de voir et de faire. Tandis que nombre de travaux avaient insisté sur les souffrances de I'exil $^{24}$, le déchirement, la double absence ${ }^{25}$, I'ici ou là-bas, les pratiques - entre autres transnationales - de pluralisme constatées témoignent d'une autre manière de vivre à la fois ici et là-bas, dans un rapport plus serein aux deux pays, et en profitant des ressources que procure chacun d'eux. Pour l'immigration maghrébine en France, la période actuelle ne se caractérise pas uniquement par son caractère colonial ou postcolonial. Si nombre de gestes politiques et des aspects des politiques publiques peuvent, selon certains auteurs, tendre à maintenir et renouveler la domination coloniale sur les pays du Maghreb (Blanchard et Bancel, 2007), si l'action publique vis-à-vis des migrants et de leurs héritiers repose toujours en partie sur des imaginaires associés à cette période (ibid.); les populations concernées construisent par leurs pratiques des relations denses, pragmatiques, routinières, débarrassées de considérations mémorielles et politiques entre la France et le Maghreb.

24 II ne s'agit pas pour nous de nier cette réalité maintes fois attestée par les cliniciens qui soignent les migrants et que deux de nos interlocuteurs ont manifesté mais d'évoquer une autre réalité, tout autant observable.

25 À propos de la condition de l'émigré-immigré, Sayad (1999, 115) conclut à son "ubiquité impossible : continuer à être présent même absent et là où on est absent (c'est le sort de l'émigré) et, corrélativement, ne pas être totalement présent, ce qui revient à y être partiellement absent ". 


\section{Références bibliographiques}

Augé Marc et Herzlich Claudine (1987) (Éds.) Le sens du mal. Anthropologie, histoire, sociologie de la maladie, Paris, Montreux, Éditions des Archives contemporaines, $278 \mathrm{p}$.

Avenel Cyprien (2009) La construction du "problème des banlieues" entre ségrégation et stigmatisation, Journal français de psychiatrie, 34, pp. 36-44.

Baubet Thierry et Moro Marie-Rose (2003) Psychiatrie et migrations, Paris, Masson, $236 \mathrm{p}$.

Bellakhdar Jamal (1997) La pharmacopée marocaine traditionnelle. Médecine arabe ancienne et savoirs populaires, Paris, Casablanca, Le Fennec, Ibis press, $764 \mathrm{p}$.

Bennani Jalil (1996) La psychanalyse au pays des saints, Casablanca, Le Fennec, $252 \mathrm{p}$.

Benoist Jean (1996) Soigner au pluriel. Essais sur le pluralisme médical, Paris, Karthala, 520 p.

Bensaâd Ali (Dir.) (2009) Le Maghreb à l'épreuve des migrations subsahariennes. Immigration sur émigration, Paris, Karthala, 448 p.

Bidet Jennifer et Wagner Lauren (2012) Vacances au bled et appartenances diasporiques des descendants d'immigrés algériens et marocains en France, Tracés. Revue de Sciences humaines, 12 (23), pp. 113-130.

Blanchard Pascal et Bancel Nicolas (2007) Culture postcoloniale 1961-2006, Paris, Autrement, $288 \mathrm{p}$.

Burns Jonathan et Tomita Andrew (2015) Traditional and religious healers in the pathway to care for people with mental disorders in Africa: a systematic review and meta-analysis, Soc Psychiatry Psychiatr Epidemiol, 50 (6), pp. 867-877.

Cant Sarah et Sharma Ursula (1999) A New Medical Pluralism? Alternative medicine, doctors, patients and the state, UCL Press, London, $226 \mathrm{p}$.

Champion Françoise (2013) Introduction. Des relations entre "religieux" et "psy", Archives de sciences sociales des religions, 3 (163), pp. 9-16.

Cherak Feza (2013) La thérapeutique de la rouqiya entre Algérie, Égypte et France, in Anne-Marie Moulin, Islam et révolutions médicales, Karthala, pp. 297-328.

Chlyeh Abdelhafid (1997) Les Gnaouas du Maroc. Itinéraires thérapeutiques, transe et possession, Paris, La Pensée Sauvage, 158 p.

Cogat-Droulez Sandrine (2001) Religion, science et "miracles", le cas de Lourdes, Socio-anthropologie, 10, [en ligne]. URL : https://journals.openedition. org/socio-anthropologie/160

Cohen Patrice et Rossi llario (2011) Le pluralisme thérapeutique en mouvement, Anthropologie \& Santé, 2, [en ligne]. URL : https://journals.openedition.org/ anthropologiesante/606

Collet Beate et Santelli Emmanuelle (2012) Le mariage "halal", réinterprétation des rites du mariage musulman dans le contexte post-migratoire français, Recherches familiales, 9, pp. 83-92. 
Connell John (2015) From medical tourism to transnational health care? An epilogue for the future, Social Science \& Medicine, 124, pp. 398-401.

Cuche Denys (2012) Migration internationales et transformation des cultures d'origine, in Chantal Crenn et Laurence Kotobi, Du point de vue de l'ethnicité, Paris, Armand Colin, pp. 43-55.

Douville Olivier (2006) Le travail clinique avec des enfants et adolescents issus de la migration, Champs, psychopathologies et clinique sociale, 2, pp. 11-20.

Dozon Jean-Pierre (1983) L'anthropologie médicale : fabrication d'un nouveau regard (Commentaire), Sciences sociales et santé, 1 (3-4), pp. 37-40.

Epelboin Alain (1996) Possession par des djinns en région parisienne, in Jean Benoist, Soigner au pluriel. Essais sur le pluralisme médical, Paris, Karthala, pp. 391-424.

Fassin Didier (Coord.) (2000a) Santé, le traitement de la différence, Hommes \& Migrations, 1225, $163 \mathrm{p}$.

Fassin Didier (2000b) Les politiques de l'ethnopsychiatrie. La psyché africaine, des colonies britanniques aux banlieues parisiennes, L'Homme, 153, pp. 231-250.

Favret-Saada Jeanne (1977) Les mots, la mort, les sorts, Paris, Gallimard, 432 p.

Gabarro Céline (2018) L'accès aux soins des étrangers en situation irrégulière en France : une prise en charge au titre de la pauvreté et de la compassion, Migrations Société, 171 (1), pp. 93-104.

Green Gill et al. (2006) "We are not completely Westernised": Dual medical systems and pathways to health care among Chinese migrant women in England, Social Science \& Medicine, 62, pp. 1498-1509.

Hammouche Abdelhafid (2007) Les recompositions culturelles. Sociologie des dynamiques sociales en situation migratoire, Strasbourg, Presses universitaires de Strasbourg, $224 \mathrm{p}$.

Hargreaves Alec et Mattelart Tristan (2012) Médias et migrations dans le bassin méditerranéen. L'internationalisation des savoirs?, Questions de communication, 21, pp. 145-156.

Hoyez Anne-Cécile (2012) "L'ayurveda, c'est pour les Français ». Interroger recours aux soins, systèmes de santé et expérience migratoire, Revue Européenne des Migrations Internationales, 28 (2), pp. 149-170.

Hoyez Anne-Cécile (2011) L'accès aux soins des migrants en France et la «culture de l'initiative locale ". Une analyse des contextes locaux à l'épreuve des orientations nationales, Cybergeo : European Journal of Geography, 566, [en ligne]. URL : https://journals.openedition.org/cybergeo/24796

Izambert Caroline (2018) La santé des immigré·e-s entre réponse médicale et approche sécuritaire, Mouvements, 93 (1), pp. 51-59.

Kepel Gilles (2012) Quatre-vingt-treize, Paris, Gallimard, 336 p.

Kessler-Bllthauer Déborah (2018) Sur le divan des guérisseurs... et des autres. À quels soins se vouer? Paris, Éditions des Archives contemporaines, 174 p.

Khosrokhavar Fahrad (1997) L'islam des jeunes, Paris, Flammarion, 319 p. 
Khrouz Nadia et Lanza Nazarena (Dirs.) (2015) Cosmopolitisme, présence d'étrangers et transformations sociales, Centre Jacques-Berque, 184 p.

Kleinman Arthur (1980) Patients and Healers in the Context of Culture. An Exploration of the Borderland between Anthropology, Medicine and Psychiatry, Berkeley, University of California Press, 448 p.

Kuczynski Liliane (2008) Attachement, blocage, blindage, Cahiers d'études africaines, 189-190, [en ligne]. URL : https://www.cairn.info/revue-cahiers-d-etudesafricaines-2008-1-page-237.htm?contenu=resume

Lacoste-Dujardin Camille (1996) Des mères contre les femmes. Maternité et patriarcat au Maghreb, Paris, La Découverte, 364 p.

Laplantine François (1986) Anthropologie de la maladie, Paris, Payot, 411 p.

Laplantine François (1982) La Maladie, la guérison et le sacré, Archives de sciences sociales des religions, 1 (54), pp. 63-76.

Laplantine François (1978) La médecine populaire des campagnes françaises aujourd'hui, Paris, J-P. Delarge, $234 \mathrm{p}$.

Le Gall Josiane et Montgomery Catherine (2009) Itinéraires thérapeutiques des nouveaux arrivants du Maghreb au Québec : entre le local et le transnational, Santé et mobilités au Nord et au Sud, Colloque AMADES, [en ligne]. URL : https://amades.hypotheses.org/1444

Lemonnier Clara (2016) Confidences féminines et sorcellerie : une ethnologie des émotions en terrain sensible, Parcours anthropologiques, 11, [en ligne]. URL : https://journals.openedition.org/pa/474

Lenclud Gérard (1987) La tradition n'est plus ce qu'elle était... Sur la notion de "tradition" et de "société traditionnelle" en ethnologie, Terrain, 9, pp. 110-123.

Lepoutre David (1997) Cour de banlieue. Codes, rites et langages, Paris, Odile Jacob, $362 \mathrm{p}$.

Loux Françoise (1974) Transmission culturelle chez les catholiques et les protestants: les soins corporels à Chardonneret, Ethnologie française, 4 (1-2), pp. $145-178$.

Ma Mung Emmanuel et. al. (1998) Bilan des travaux sur la circulation migratoire : Rapport final, commandé par le ministère de la Solidarité et de I'Emploi, Direction de la Population et des Migrations (DPM), Paris, 148 p.

Marcellini Anne, Turpin Jean-Philippe, Rolland Yannick et Ruffié Sébastien (2000) Itinéraires thérapeutiques dans la société contemporaine, Corps et culture, 5, [en ligne]. URL : https://journals.openedition.org/corpsetculture/710

Monteilet Nicolas (2005) Le pluralisme thérapeutique au Gabon, Paris, Karthala, $262 \mathrm{p}$.

Moro Marie-Rose et Baubet Thierry (2013) Psychopathologie transculturelle, Paris, Masson, $297 \mathrm{p}$.

Nathan Tobie (1994) L'influence qui guérit, Paris, Odile Jacob, 352 p.

Palazzolo Jérome, Midolb Nancy et Candau Joël (2008) Vers une gestion autonome de la médication en psychiatrie? Approche anthropologique, Annales Médico-psychologiques, 166 (9), pp. 717-726. 
Pordié Laurent (2011) Se démarquer dans l'industrie du bien-être. Transnationalisme, innovation et indianité, Anthropologie \& Santé, 3, [en ligne]. URL : https://journals.openedition.org/anthropologiesante/805\#quotation

Raineau Clémentine (2002) Maladie et infortune dans l'Auvergne d'aujourd'hui. Médecins, malades et guérisseurs d'un bourg montagnard à l'hôpital, Ruralia, 10/11, [en ligne]. URL : https://journals.openedition.org/ruralia/309

Razy Élodie et Baby-Collin Virginie (2011) La famille transnationale dans tous ses états, Autrepart, 1 (57-58), pp. 7-22.

Rechtman Richard (2010) La psychiatrie à l'épreuve de l'altérité. Perspectives historiques et enjeux actuels, in Didier Fassin Dir., Les Nouvelles Frontières de la société française, Paris, La Découverte, pp. 101-127.

Rechtman Richard (2000) De la psychiatrie des migrants au culturalisme des ethnopsychiatres, Hommes et Migrations, 1225, pp. 46-61.

Sakoyan Juliette (2008) Approche anthropologique de la représentation de l'autisme en situation migratoire : les mères d'origine comorienne confrontées à I'autisme, L'Information Psychiatrique, 81 (4), pp. 325-332.

Sakoyan Juliette, Musso Sandrine et Mulot Stéphanie (2011) Quand la santé et les médecines circulent, Anthropologie \& Santé, 3, pp. 1-13.

Sayad Abdelmalek (1999) La Double Absence. Des illusions de l'émigré aux souffrances de l'immigré, Paris, Seuil, $448 \mathrm{p}$.

Schmitz Olivier (Éd.) (2006) Les médecines en parallèle - Multiplicité des recours au soin en Occident, Paris, Karthala, 278 p.

Sciences sociales et santé (1985) Anthropologie, sociétés et santé, Sciences sociales et santé, 3 (3-4), 207 p.

Sicot François et Touhami Slimane (2015) Les professionnels français de la santé mentale et la culture de leurs patients étrangers ou d'origine étrangère, Anthropologie et santé, 10, [en ligne]. URL : https://journals.openedition.org/ anthropologiesante/1725

Sindzingre Nicole (1985) Présentation : tradition et biomédecine, Sciences sociales et santé, 3 (3-4), pp. 9-26.

Stan Sabina (2015) Transnational healthcare practices of Romanian migrants in Ireland: Inequalities of access and the privatisation of healthcare services in Europe, Social Science \& Medicine, 124, pp. 346-355.

Streiff-Fenart Jocelyne (2006) À propos des valeurs en situation d'immigration : questions de recherche et bilan des travaux, Revue française de sociologie, 47 (4) pp. 851-875.

Streiff-Fenart Jocelyne et Poutignat Philippe (1995) Théories de l'ethnicité, Paris, Presses Universitaires de France, $270 \mathrm{p}$.

Tarrius Alain (1993) Territoires circulatoires et espaces urbains : différentiation des groupes migrants, Les Annales de la recherche urbaine, 59-60, pp. 51-60.

Tillon Germaine (1966) Le Harem et les cousins, Paris, Le Seuil, 219 p.

Touag Hanifa (2012) Guérir par l'islam : I'adoption du rite prophétique - Roqya - par les salafistes en France et en Belgique, in Brigitte Maréchal et Farid Asri, Islam belge au pluriel, Louvain, PUL, pp. 201-218. 
Touhami Slimane (2010) La part de I'œil. Une ethnologie du Maghreb de France, Paris, CTHS, 279 p.

Wang Simeng (2017) Illusions et souffrances. Les migrants chinois à Paris, Paris, Rue D'Ulm Editions, 220 p.

Wang Simeng (2016) "Aidez-nous à comprendre vos Chinois!». Conditions de possibilités de la légitimation du sociologue en milieu psychiatrique, Genèses, 4 (105), pp. 141-156. 


\section{François Sicot et Slimane Touhami}

\section{Le pluralisme thérapeutique des migrants et héritiers de l'exil maghrébin en France. Nouvelles données et perspectives}

Les itinéraires de soins des patients maghrébins adoptent souvent des formes de pluralisme médical. Ces patients, migrants ou nés en France, recourent en effet pour bon nombre à des soins traditionnels au Maghreb (fqih, roqya, prière, désensorceleur). L'existence de ces pratiques s'explique tout autant par le rapport entretenu avec les cultures du pays d'émigration et la trajectoire sociale que par le temps passé dans le pays d'accueil ou l'expérience migratoire. Les patients et leurs proches ne sont pas ancrés dans une culture d'origine qui expliquerait leurs recours, car ils n'impliquent pas la croyance ou une adhésion pleine et entière à une étiologie des troubles mentaux. Une offre disponible, facile d'accès ainsi que des insatisfactions vis-à-vis de la psychiatrie expliquent le pragmatisme dont font preuve les patients et leurs proches en recourant à une diversité de soins. Les recours traditionnels peuvent être réalisés en France ou lors d'un retour au pays d'origine, ici et là-bas. Mais lors de leurs retours au pays, les patients maghrébins peuvent également consulter des psychiatres dont ils apprécient l'empathie et le partage de référentiels communs. Le pluralisme médical des patients maghrébins en France représente une modalité de l'expérience transnationale.

\section{Therapeutic Pluralism of Migrants and Heirs of the Maghreb Exile in France. New Data and Perspectives}

Treatment of Maghreb patients is characterised by the practice of medical pluralism. Many of these patients, immigrants or born in France, resort to practices popular in the Maghreb (fqih, roqya, prayer and dewitching). The existence of these practices can be explained as much by maintaining the culture of the country of immigration and the social trajectory as by the time spent in the host country or the migration experience. The patients and their relatives are not anchored in a culture of origin, which explains their actions as they do not believe in or fully adhere to the aetiology of mental disorders. An available solution, easy to access, and dissatisfaction of psychiatry explain the pragmatism demonstrated by patients and their relatives who use a variety of treatments. Popular remedies may be carried out in France or when returning to the country of origin where Maghreb patients may also consult psychiatrists with whom they share cultural sensitivity. Medical pluralism of Maghreb patients in France represents a transnational modality. 


\section{El pluralismo terapéutico de los migrantes y descendientes del exilio magrebí en Francia. Nuevos hechos y perspectivas}

Los itinerarios de atención de pacientes magrebíes se caracterizan por la práctica del pluralismo médico. Estos pacientes, inmigrantes o nacidos en Francia, recurren a menudo para tratarse mediante una atención popular en el Magreb (fqih, roqya, oración, deshechizado). La existencia de dichas prácticas se explica a la vez por la relación que se mantiene con la cultura del país de emigración y por la trayectoria social, así como por el tiempo pasado en el país de acogida o en relación a la experiencia migratoria. Los pacientes y sus parientes u otras relaciones cercanas no están ancladas en una cultura de origen que explicaría sus medios, puesto que no implican la creencia o una adhesión plena o completa a una etiología de los trastornos mentales. Una oferta disponible, de fácil acceso, añadida a una insatisfacción respecto a la psiquiatría, explican el pragmatismo que los pacientes y sus relaciones cercanas experimentan recurriendo a una diversidad de atenciones. Las intervenciones populares se pueden realizar en Francia o durante un regreso al país de origen, aquí y allí. Aunque durante la vuelta al país, los pacientes magrebíes pueden a su vez consultar a psiquiatras de quienes aprecian su sensibilidad cultural. El pluralismo médico de los pacientes magrebíes en Francia representa una forma o práctica transnacional. 